\title{
Serotonin Depletion Does Not Prevent Intrinsic Sensitization in the Leech
}

\author{
Brian D. Burrell ${ }^{1}$ and Christie L. Sahley \\ Department of Biological Sciences \\ Purdue University \\ West Lafayette, Indiana 47907 USA
}

\begin{abstract}
Intrinsic sensitization is a form of behavioral facilitation that is distinct from the extrinsic sensitization normally studied. To examine whether intrinsic and extrinsic sensitization are mediated by different physiological processes, the effects of 5,7-dihydroxytryptamine-induced serotonin (5-HT) depletion on intrinsic sensitization of the leech whole-body shortening response were observed. Previous experiments have shown that 5-HT depletion disrupts dishabituation and extrinsic sensitization of this behavior in the leech. Intrinsic sensitization was observed in preparations from both control and 5-HT-depleted animals, indicating that this form of behavioral facilitation was not affected by 5-HT depletion. The differences in the effects of 5-HT depletion on intrinsic versus extrinsic sensitization suggest that there are distinct neurophysiological processes mediating these two forms of behavioral facilitation. In addition, 5-HT depletion appeared to disrupt a putative extrinsic form of habituation of the shortening reflex. These data support the hypothesis that both intrinsic and extrinsic processes of neuromodulation mediate habituation and sensitization.
\end{abstract}

\section{Introduction}

Sensitization is a form of non-associative learning involving the facilitation of a given test behavior following the presentation of a sensitizing

\footnotetext{
${ }^{1}$ Corresponding author. Present address: Department of Physiology \& Biophysics, University of Miami School of Medicine, Miami, Florida 33136 USA.
}

stimulus (Thompson et al. 1973). This sensitizing stimulus usually differs from the stimulus used to elicit the test behavior in terms of either intensity or modality. Once sensitization is induced, potentiation of the test behavior is observed throughout the body, regardless of where or what kind of test stimulus is applied (Castellucci and Kandel 1976; Erber and Schildberger 1980; Boulis and Sahley 1988; Davis 1989). One caveat of this generalization property is that sensitization will only affect behaviors of a similar category. For example, a noxious stimulus can sensitize aversive behavior, e.g., a defensive withdrawal response, and an appetitive stimulus can sensitize the appropriate appetitive behavior, e.g., exposure to food sensitizing a feeding response, but an aversive stimulus cannot sensitize an appetitive behavior or vice versa (Kupferman and Pinsker 1968; Advocat 1980). This form of non-associative learning is of interest because of evidence that the physiological processes that underlie sensitization also contribute to associative learning (Hawkins et al. 1993; Sahley 1994).

Using the leech whole-body shortening reflex, we have observed a form of behavioral facilitation that resembles sensitization, but has properties that make it distinct from "normal" sensitization (Burrell and Sahley 1998). In brief, two mechanosensory stimuli were used to test the shortening response, a novel $\left(\mathrm{S}_{\mathrm{N}}\right)$ and training $\left(\mathrm{S}_{\mathrm{T}}\right)$ stimulus. These stimuli were approximately equivalent in intensity and differ only in where the stimuli were applied in a single segment of leech skin. During training, each preparation was initially stimulated at the $S_{N}$ to obtain a baseline shortening response for that site. Shortening at the $S_{T}$ was then induced in a repetitive manner that normally induces habituation of this response. This was followed by a second test of $\mathrm{S}_{\mathrm{N}}$-elicited shortening. In preparations where the $S_{T}$ and $S_{N}$ were relatively distant from each other (dorsal vs. ventral sides of the body segment) the results were not surprising, the

LEARNING \& MEMORY 6:509-520 @ 1999 by Cold Spring Harbor Laboratory Press ISSN1072-0502/99 \$5.00

$$
\begin{array}{lllllllllllllll}
L & E & A & R & N & I & N & G & \underset{509}{\mathbf{2}} & M & E & M & O & R & Y
\end{array}
$$


$\mathrm{S}_{\mathrm{T}}$-induced shortening habituates while the $\mathrm{S}_{\mathrm{N}}$ behavior is unchanged from its initial baseline. However, in preparations where the $S_{T}$ and $S_{N}$ were close together (on the same side of the body segment), $\mathrm{S}_{\mathrm{T}}$-induced shortening did not habituate following repetitive stimulation, but $\mathrm{S}_{\mathrm{N}}$-elicited responses were significantly lower than their initial baseline response. That is, the site that directly received habituating stimuli exhibited no behavioral decrement, while a second, nearby site that did not receive habituating stimuli did experience a response decrement. Both the facilitation observed at the $S_{\mathrm{T}}$ and the response decrement at the $\mathrm{S}_{\mathrm{N}}$ were dependent on the proximity of these two stimulation sites to each other. The selective facilitation at the $S_{\mathrm{T}}$ was unlikely to be due to short-term processes such as paired-pulse facilitation or posttetanic potentiation since the behavioral facilitation persisted for the $40 \mathrm{~min}$ duration of habituation training. In addition, facilitation at the $S_{T}$ was still observed when a $10 \mathrm{~min}$ resting period was interposed between the initial $S_{N}$ stimulation and the beginning of habituation training at the $\mathrm{S}_{\mathrm{T}}$ (Burrell and Sahley 1998).

The behavioral facilitation at the $S_{\mathrm{T}}$ resembles sensitization of the leech shortening response in that it prevents the normal behavioral decrement following habituation training (Boulis and Sahley 1988) but differs from this form of sensitization in two critical ways. First, the observed behavioral facilitation was induced by a stimulus (the $S_{N}$ ) that was very similar to the $S_{T}$, both in terms of intensity and position on the skin where the stimulus was applied (Burrell and Sahley 1998). Second, the observed behavioral facilitation was limited to a specific stimulus-response pathway (the $\mathrm{S}_{\mathrm{T}}$ ) and was not observed at the $S_{N}$, which appeared to be habituated due to the repetitive stimulation at the $\mathrm{S}_{\mathrm{T}}$. This occurred even though the $\mathrm{S}_{\mathrm{T}}$ and $\mathrm{S}_{\mathrm{N}}$ were very similar in terms of the sensory cells shared by both stimulation sites.

It was concluded that this behavioral plasticity represented an intrinsic sensitization process, using the terminology from Davis and File (1984). Intrinsic sensitization, also known as incremental (Razran 1971) or wind-up sensitization (Hinde 1970), describes a process in which the physiological mechanisms that potentiate the test behavior occur entirely within the stimulus-response pathway of that very behavior. This differs from extrinsic sensitization, where the facilitation is dependent on the contributions from neural elements outside of the test behavior's stimulus-response pathway (Thompson et al. 1973; Castellucci and Kandel 1976; Hitchcock et al. 1989; Hawkins et al. 1993). Intrinsic sensitization has been observed in a variety of vertebrates and invertebrates including the leech (Lockery and Kristan 1991; Burrell and Sahley 1998), the mollusk Helix aspera (Prescott and Chase 1996), the cat (Groves et al. 1970), the rat (Davis and Wagner 1969; Schanbacher et al. 1996), and in human infants (Bashinski et al. 1985).

Evidence of physiological processes contributing to extrinsic sensitization are found in Aplysia, where activation of serotonergic interneurons results in heterosynaptic facilitation of sensory-tomotor/interneuron synapses, which contributes to behavioral sensitization (Hawkins et al. 1993). Sensitization of leech shortening is also dependent on serotonergic neurons, specifically the Retzius (R) cells (Ehrlich et al. 1992; Sahley 1994). If sensitization is truly mediated by distinctive intrinsic and extrinsic mechanisms, then different physiological processes should mediate each type of sensitization. In the leech, depletion of serotonin (5-HT) from the R cells using the 5-HT analog, 5,7-dihydroxytryptamine (5,7-DHT), prevents sensitization of the whole-body shortening response (Ehrlich et al. 1992; Sahley 1994). In Aplysia, 5,7-DHT-induced 5-HT depletion disrupts dishabituation and prevents the heterosynaptic facilitation produced by dishabituating stimuli (both sensitization and dishabituation are produced by the same noxious stimulus) (Glanzman et al. 1989). Assuming that 5-HT-dependent sensitization is extrinsic in nature, it is reasonable to hypothesize that intrinsic sensitization should not be affected by manipulations that disrupt extrinsic sensitization. Therefore, we used 5-HT-depletion techniques and intrinsic sensitization training procedures to assess the role of 5-HT on intrinsic sensitization. We confirmed the hypothesis that intrinsic sensitization in the leech was not dependent on serotonergic modulation.

\section{Materials and Methods}

\section{5-HT DEPLETION}

For these experiments, 3-gram leeches were obtained from a commercial supplier (Leeches USA) and maintained in artificial pond water (1 gram/2 liter $\mathrm{dH}_{2} \mathrm{O}$ Hirudo Salt, Leeches USA) in a $20^{\circ} \mathrm{C}$ room. All dissections were carried out in leech saline (Muller et al. 1981) maintained at $\sim 4^{\circ} \mathrm{C}$. The protocol for 5-HT depletion in the leech was the same as that used by Ehrlich et al. (1992).

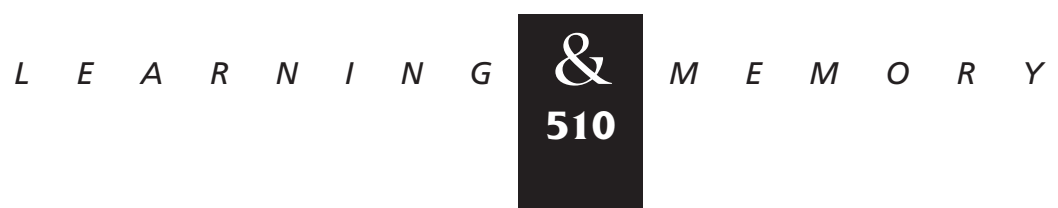


The ventral blood sinus, which contains the leech nerve cord, was exposed and a small incision was made. A fixed volume $(0.1 \mathrm{ml})$ of $1.0 \mathrm{~mm}$ 5,7-DHT (creatine sulfate complex, Sigma) dissolved in 65 mu ascorbic acid saline was injected into the ventral blood sinus of animals in the 5-HT-depleted group. A second group of animals (control group) received injections of ascorbic acid saline solution. At least two weeks were allowed to pass before the animals underwent behavioral testing to allow time for recovery from the injection procedure. No animals were used beyond one month after treatment, which insured that all leeches were tested during a time of maximum 5-HT depletion (Ehrlich et al. 1992).

\section{BEHAVIORAL TESTING PROTOCOL}

All behavioral experiments were conducted using the leech quasi-intact preparation (Burrell and Sahley 1998). Each preparation was implanted with two pairs of $0.2-\mathrm{mm}$ Teflon-coated silver wire (Medwire) electrodes, bared at the point of contact to stimulate discrete areas of the leech skin. The electrodes in each pair were bared enough so that three annuli within a segment (five annuli/segment) were in contact with the exposed wire. One pair of electrodes was designated the $S_{\mathrm{T}}$ (trained site) and was used for habituation training. A second pair of electrodes was designated the $S_{N}$ (novel site) and would be used to test for generalization of habituation. Both electrode pairs were implanted in the same body segment (segment 8), very close together so that they activated approximately the same set of mechanosensory cells. Ganglia in segments 7-9 of the preparation were exposed so that individual neurons could be recorded from intracellularly.

The shortening response was stimulated via the delivery of a single, 2 msec duration, capacity coupled electroshock to the skin with the stimulation parameters controlled by a Grass S88 Stimulator and SIU5 Stimulus Isolation Unit. The intensity of the shock was set to the behavioral threshold of each preparation. Intrinsic sensitization training was carried out in the following manner on preparations in both the control and 5-HT-depleted groups. Before repetitive stimulation at the $\mathrm{S}_{\mathrm{T}}$, the $\mathrm{S}_{\mathrm{N}}$ was stimulated [three pulses for $2 \mathrm{~min}$ intertrial interval (ITI)]. This stimulation protocol was observed in previous experiments to induce intrinsic sensitization of the leech shortening response (Burrell and Sahley 1998) and also provided a baseline response level for $\mathrm{S}_{\mathrm{N}}$-elicited shortening. Two min- utes after the last the $S_{N}$ baseline trial, habituation training was carried out at the $\mathrm{S}_{\mathrm{T}}$ site using the protocol outline by Sahley et al. [(1994) 20 trials, one pulse per trial for 2-min ITI]. Data from the 20 habituation-training trials were averaged into four 5-trial blocks. The first block (consisting of trials 1-5) was used as the baseline for the subsequent responses elicited at the $S_{\mathrm{T}}$. After habituation training at the $S_{T}$, the behavioral response at the $S_{N}$ site was probed for generalization of habituation (as with the baseline, the average response across three trials). A third group of preparations (habituation-only) had only habituation training carried out on them and did not receive the extra stimuli at the $S_{N}$ associated with intrinsic sensitization and did not receive an injection of ascorbic acid or 5,7-DHT.

The magnitude of an evoked shortening response was measured using an isometric tension transducer (Grass Instruments). Extracellular recordings were made using a Grass P/5 A.C. preamplifier. Intracellular recordings of the $\mathrm{R}$ cells were made using sharp glass microelectrodes (15$20 \mathrm{~m} \lambda$ resistance; Sutter Instruments P-80 micropipette puller) filled with $3 \mathrm{~m}$ potassium acetate and an Axoclamp 2A amplifier (Axon Instruments). Data from recording devices was viewed on a storage oscilloscope (Tektronix) and converted for digital storage using a TL-1 analog/digital interface and pClamp 6.21 data acquisition program (Axon Instruments).

\section{NEUROANATOMY PROCEDURES}

Following behavioral training, the nerve cords were dissected from the preparation and tested for the presence of 5-HT using a modified glyoxylic acid staining technique (Macagno and Stewart 1987). The tissue was incubated in the glyoxylic acid solution $(68 \mathrm{mg} / \mathrm{ml}$ sucrose, $32 \mathrm{mg} / \mathrm{ml} \mathrm{mono-}$ basic potassium phosphate, $10 \mathrm{mg} / \mathrm{ml}$ glyoxylic acid at $\mathrm{pH} 7.4$ ) for $5 \mathrm{~min}$ and then allowed to airdry 20-30 min. A drop of mineral oil was added to the dried tissue, which was then mounted on a microscope slide in mineral oil and heated at $95^{\circ} \mathrm{C}$ for $5 \mathrm{~min}$. The stained ganglia were viewed using a fluorescent microscope with a UV filter and imaged with a digital camera and computer imaging software (Metamorph).

\section{STATISTICS}

During data analysis, the behavioral data from each trial block was normalized to the initial base-

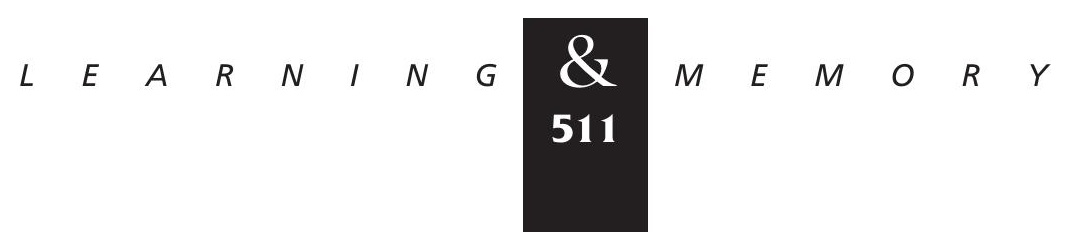


line response. In the case of the $\mathrm{S}_{\mathrm{N}}$, data from the post-habituation trial block were normalized to the baseline trial block taken before habituation training at the $S_{\mathrm{T}}$. In the case of the $\mathrm{S}_{\mathrm{T}}$, the habituation trial blocks (trial block 2-4) were normalized to trial block 1. Behavioral data were analyzed using one-way and two-way analysis of variance (ANOVA) with repeated measures. Analysis was carried out using the Statistica (Statsoft, Inc.) software package.

\section{Results}

\section{5-HT DEPLETION}

Based on the glyoxylic acid staining results (Fig. 1), the 5,7-DHT treatments in these experiments depleted 5-HT from the leech CNS to a similar extent as observed in previous experiments (Ehrlich et al. 1992; Sahley 1994). Ganglia from control group animals exhibited fluorescent staining in the $\mathrm{R}$ cells, the posteromedial (M), the ventrolateral (VL), and dorsolateral (DL) cells (Fig. 1A). Ganglia from 5,7-DHT-treated animals showed no fluorescence in the R cells, while the VL, DL, and M cells were still positively stained (Fig. 1B). Furthermore, $\mathrm{R}$ cells in the depleted group exhibited a brown pigmentation that was also visible under normal light (data not shown). In agreement with Lent (1984), R cells in the depleted group were not dead based on the fact that they retained their normal membrane resting potential and the ability to generate action potentials (data not shown).
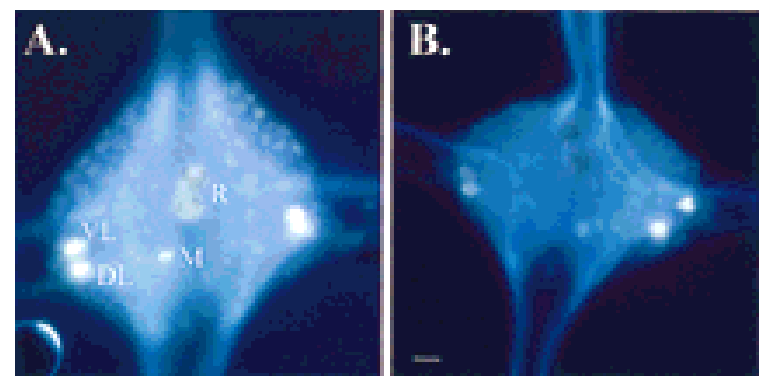

Figure 1: Glyoxylic acid staining of 5-HT-depleted and control ganglia. (A) Ganglion from a leech treated with ascorbic acid. In these control animals, the $\mathrm{R}$ cells do stain positive for 5 -HT. Other serotonergic neurons that are stained by this procedure are the $\mathrm{DL}, \mathrm{VL}$, and posteromedial (M) neurons. (B) Ganglion from a leech treated with 5,7-DHT. Although other 5-HT-containing neurons still stain positive for $5-\mathrm{HT}$, the $\mathrm{R}$ cells do not stain for 5-HT indicating depletion of 5-HT. Bar, $50 \mu \mathrm{m}$.

\section{BEHAVIORAL RESULTS}

Following stimulation at the $\mathrm{S}_{\mathrm{N}}$, the wholebody shortening response elicited at the $S_{\mathrm{T}}$ did not significantly decrease in magnitude during habituation training in either the control or 5-HT-depleted groups when compared to the group of preparations that underwent normal habituation training (no stimulation at a second novel site prior to training; Fig. 2B). A two-way ANOVA demonstrated a significant effect of treatment group (control, 5-HT-depleted, and habituationonly groups; $F_{\text {treat }}=4.45, P \leq 0.05, \mathrm{df}=42$ ) and training (trial blocks $2-4 ; F_{\text {trial }}=5.09, P \leq 0.05$, df $=84)$, but no treatment/trial block interaction effect $\left(F_{\text {inter }}=2.03, P>0.05, \mathrm{df}=84\right)$. Post hoc analysis (Neuman-Keuls) for the effect of treatment group showed that while shortening in the control and the 5-HT-depleted groups were both significantly different from responses in the habituationonly group $(P \leq 0.05)$, they were not different from each other $(P>0.05)$. These results indicate that both control and 5-HT-depleted leeches were capable of undergoing intrinsic sensitization at the $S_{\mathrm{T}}$ due to stimulation at the $S_{\mathrm{N}}$ prior to the beginning of habituation training. They also indicate that the magnitude of the shortening response in groups that underwent intrinsic sensitization training was significantly greater than in preparations from the habituation-only group.

The statistically significant effect of trial block reflects the relative decrease in the shortening response between trial blocks 2 and 4 . However, as Fig. 2B shows, the actual decrease in the shortening response relative to the initial response level in the control and 5-HT-depleted groups is much less than in the habituation-only group. Earlier experiments (Burrell and Sahley 1998) clearly show that preparations that had undergone the same intrinsic sensitization training as the control and 5-HT-depleted groups did not habituate. The lack of significant treatment/trial block interaction effect in the current data set did not allow a post-hoc test of effects of trials to be carried out on each of the treatment groups. Instead a one-way ANOVA, testing for differences between the three treatment groups, was carried out on the shortening responses from trial block 4 only. Because all the data has been normalized to an initial baseline (trial block 1), the magnitude of the response at trial block 4 reflects the level of behavioral decrement due to habituation training. The results of this analysis demonstrate a statistically significant effect

$$
\begin{array}{lllllllll}
L & E & A & R & N & I & N & G & \mathbf{Q} \\
\mathbf{5 1 2} & M & E & M & O & R & Y
\end{array}
$$


A

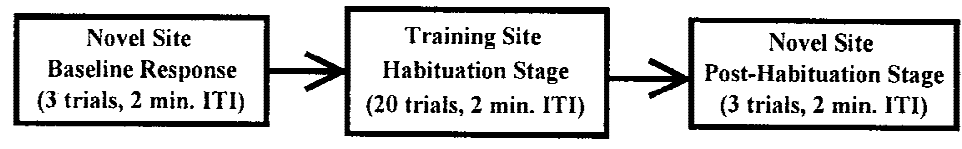

B

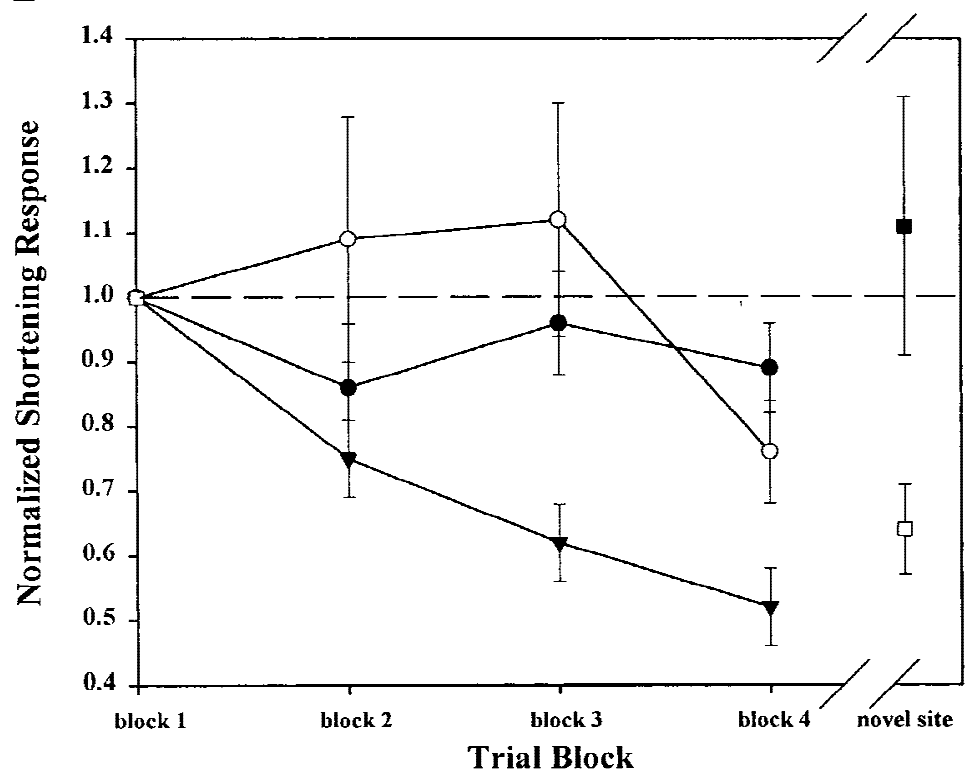

Figure 2: Effects of 5-HT depletion on intrinsic sensitization and extrinsic habituation. $(A)$ Experimental protocol for intrinsic sensitization training of the leech whole-body shortening response. This protocol was used to test preparations from both the control and 5-HTdepleted groups. Preparations from the habituation-only group did not receive mechanosensory stimuli at a second, novel site prior to or after habituation training. (B) $\mathrm{S}_{\mathrm{T}}$-elicited behavior from both the control $(\bigcirc)$ and 5-HT-depleted (-) preparations did not habituate in spite of repeated mechanosensory stimulation (trial blocks 1-4) following the induction of intrinsic sensitization. The habituation-only group $(\boldsymbol{\nabla})$ demonstrates the changes in the shortening response during normal habituation training, when intrinsic sensitization is not induced. $\mathrm{S}_{\mathrm{N}^{-}}$-elicited shortening did significantly decrease due to a putatively extrinsic form of habituation in the control group ( $\square$ ), but the $S_{N}$ response in the 5-HT-depleted group $(\mathbf{\square})$ did not habituate. of the treatment group on the trial block 4 responses $(F=7.30, P \leq 0.01)$. Post hoc analysis showed that the trial block 4 responses from both control and 5-HT-depleted groups were significantly higher than those from the habituation-only group ( $P \leq 0.05$ and $P \leq 0.01$, respectively), but were not significantly different from each other $(P>0.05)$. This analysis supports the conclusion that both the control and 5-HT-depleted groups did not undergo habituation as a result of intrinsic sensitization training.

Although 5-HT depletion did not affect intrinsic sensitization at the $S_{\mathrm{T}}$, the expression of habituation normally seen at the $S_{N}$ was surprisingly absent in the 5-HT-depleted group. In earlier experiments (Burrell and Sahley 1998), this same training procedure produced behavioral facilitation in $\mathrm{S}_{\mathrm{T}}$-elicited shortening and behavioral depression in $S_{N^{-}}$-elicited responses. This latter effect was presumably due to generalization of habituation training from the $S_{T}$ to the $S_{\mathrm{N}}$. However, in the current experiments, $\mathrm{S}_{\mathrm{N}}$-elicited shortening in preparations from the 5-HT-depleted group did not decrease relative to baseline following habituation training at the $\mathrm{S}_{\mathrm{T}}$ (Fig. 2B). The $\mathrm{S}_{\mathrm{N}}$ responses from the control group, on the other hand, were substantially lower than the initial baseline after training, in agreement with earlier observations (Burrell and Sahley 1998). A two-way ANOVA confirmed that 5-HT depletion had a significant effect on $\mathrm{S}_{\mathrm{N}}$-elicited behavior following $\mathrm{S}_{\mathrm{T}}$ habituation training $\left(F_{\text {treat }}=4.25, \quad P \leq 0.05, \quad\right.$ df $=29$; $F_{\text {trial }}=1.23, P>0.05, \mathrm{df}=29 ; F_{\text {inter }}=4.25, P \leq 0.05$, $\mathrm{df}=29$ ). Post hoc analysis of these results showed that $\mathrm{S}_{\mathrm{N}}$-elicited shortening in the control group was significantly lower then the responses before training $(P \leq 0.05)$, while $\mathrm{S}_{\mathrm{N}}$ behavior from the 5 -HTdepleted group was unchanged from baseline $(P>0.05)$.

The injections of 5,7-DHT or ascorbic acid did not alter whole-body shortening itself. A comparison of tension transducer measurements taken during trial block 1 showed no statistical differences between the 5-HT-depleted, the control (ascorbic acid injected) or the habituation-only groups (means of tension transducer recordings $=0.22 \mathrm{~g}$, 0.22 g, 0.29 grams, respectively; one-way ANOVA: $F=1.18, \mathrm{df}=42, P>0.05)$.

\section{DISCUSSION}

These experiments provide the first evidence that intrinsic sensitization of the leech whole-body

$$
\begin{array}{lllllllllllllll}
L & E & A & R & N & I & N & G & \mathbf{8} & M & E & M & O & R & Y \\
\mathbf{5 1 3} & & & & & & & &
\end{array}
$$


shortening response involves physiological mechanisms that are distinct from those of extrinsic sensitization of this behavior. Intrinsic sensitization of $\mathrm{S}_{\mathrm{T}}$-elicited shortening was equally inducible in both control and 5-HT-depleted leeches. These results suggest that intrinsic sensitization of leech shortening is a 5-HT-independent process, unlike extrinsic sensitization and dishabituation of shortening in the leech (Ehrlich et al. 1992; Sahley 1994) or dishabituation of gill withdrawal in Aplysia (Glanzman et al. 1989), both of which are disrupted by 5-HT-depletion.

Another interesting result from these experiments was that 5-HT depletion altered the habituation process in $\mathrm{S}_{\mathrm{N}}$-elicited behavior that normally occurs following habituation training at the $\mathrm{S}_{\mathrm{T}}$. This was particularly surprising, as 5-HT depletion has not been observed to affect habituation in the leech, Aplysia, or Helix locorum (Zakharov and Balaban 1987; Glanzman et al. 1989; Ehrlich et al. 1992; Sahley 1994). Nevertheless, the current data suggest that 5-HT plays a role in habituation of leech whole-body shortening.

\section{BEHAVIOR}

Based on the data presented above, 5-HT depletion does not alter the ability of the leech whole-body shortening response to undergo intrinsic sensitization. During intrinsic sensitization training, the behavioral responses of preparations that had undergone 5,7-DHT-induced 5-HT depletion did not differ from those of the ascorbic acid control groups. These results differ from previous experiments with the leech where 5-HT depletion completely disrupted extrinsic sensitization and partially disrupted dishabituation and associative learning (Ehrlich et al. 1992; Sahley 1994). They also differ from studies in Aplysia where 5-HT depletion disrupts dishabituation and prevents heterosynaptic facilitation induced by dishabituating stimuli (Glanzman et al. 1989). Even though extrinsic sensitization and dishabituation are known to be distinctive forms of learning (Rankin and Carew 1988; Sahley 1994), these results are relevant because both forms of learning are induced by the same type of stimulus and, in the case of Aplysia, involve the heterosynaptic facilitation at the same synapses (Hawkins et al. 1993). The data indicate that differences in the 5-HT-dependence between intrinsic and extrinsic sensitization in the leech reflect differences between these two forms of non- associative learning. Extrinsic sensitization is produced by stimuli that are qualitatively different from the stimuli used in this paper in terms of stimulus magnitude, position/direction, or modality (Castellucci and Kandel 1976; Boulis and Sahley 1988; Davis 1989). Stimuli that produce extrinsic sensitization potentiate behavior through the activity of extrinsic modulatory interneurons that facilitate one or more neurons involved in generating the behavior of interest (Hawkins et al. 1981; Ehrlich et al. 1992). Once the extrinsic modulatory neuron has been activated, every behavior whose underlying neural circuit is sensitive to the modulatory transmitter will be affected. Based on the results from earlier 5-HT-depletion studies in the leech, the serotonergic $\mathrm{R}$ cells appear to represent the source of extrinsic modulation that mediates extrinsic sensitization of leech whole-body shortening (Ehrlich et al. 1992; Sahley 1994).

Intrinsic sensitization, on the other hand, is dependent on modulatory processes that occur within the stimulus-response pathway (Davis and File 1984; Prescott 1998) and thus, one would not expect intrinsic sensitization to be affected by manipulations that disrupt extrinsic sensitization. As the results of this study show, intrinsic sensitization was not affected by a level of 5-HT depletion (see below) known to disrupt extrinsic sensitization in the leech. These results are in agreement with a similar series of experiments involving intrinsic sensitization of the rat acoustic startle response. Extrinsic sensitization of this reflex is known to involve modulatory input from the amygdala and lesions of this structure have been shown to disrupt extrinsic, but not intrinsic, sensitization of the startle response (Hitchcock et al. 1989; Schanbacher et al. 1996).

Evidence that the site of intrinsic sensitization is within the stimulus-response pathway is provided by behavioral results from Burrell and Sahley (1998). While the $S_{T}$ is sensitized, indicated by the fact that it does not habituate following repeated stimulation, this same repetitive stimulus does habituate the $S_{N}$ despite the fact that the $S_{T}$ and $S_{N}$ share nearly all the same sensory cells (Burrell and Sahley 1998). In fact, the closer, and therefore more similar, the "sensitizing" $\mathrm{S}_{\mathrm{N}}$ stimulus was to the $S_{\mathrm{T}}$ (which corresponded to the number of shared mechanosensory neurons), the greater the level of facilitation observed during subsequent habituation training at the $\mathrm{S}_{\mathrm{T}}$. That the facilitating process is so specific indicates that this form of sensitization is mediated by modulation within the 
stimulus-response pathway activated by the test stimulus (in this case, the $\mathrm{S}_{\mathrm{T}}$ ).

One difference between the behavioral results observed here and those from previous intrinsic sensitization experiments in the leech is that the shortening reflex elicited at the $\mathrm{S}_{\mathrm{N}}$ did not habituate in the 5-HT-depleted group. This was surprising because the same training protocol used in the current experiments reliably produced sensitization at the $S_{T}$ and habituation at the $S_{N}$ in three separate groups of animals (Burrell and Sahley 1998) as well as in the control group in the current experiment. Although habituation was not directly tested in the 5-HT-depleted leeches used in these experiments, 5-HT depletion did not affect habituation of shortening in the leech in two separate experiments using the same depletion protocol (Ehrlich et al. 1992; Sahley 1994). Similarly, habituation was not disrupted in the snail, H. lucorum, by 5,7-DHTinduced 5-HT depletion (Zakharov and Balaban 1987). The lack of habituation at $S_{N}$ can not be due to an effect on the shortening response itself because neither the control nor the 5-HT-depleted group differed in terms of initial magnitude of behavior. Therefore, the effect of 5-HT depletion on the $\mathrm{S}_{\mathrm{N}}$-elicited responses must be thought of in terms of an effect on learning and not on the shortening response itself.

A potential explanation is that $5-\mathrm{HT}$ depletion has disrupted a physiological component of habituation that could not be detected in earlier depletion experiments in the leech because of differences in training protocols between those experiments and the one used in this study. As with sensitization, it is likely that habituation is mediated by intrinsic and extrinsic processes of neuroplasticity (Davis and File 1984). The intrinsic component of habituation may involve homosynaptic depression of sensory neurons onto their post-synaptic targets as described in Aplysia (Castellucci et al. 1970; Hawkins et al. 1993). The extrinsic or heterosynaptic component of habituation could potentially involve an inhibitory neuron(s) that is not part of the actual neural circuit of a given test behavior, but is activated by repetitive stimulation and has an inhibitory effect on one or more components of that circuit. Evidence of a heterosynaptic, extrinsic component to habituation has been observed in Aplysia (Goldberg and Lukowiak 1984; Stopfer and Carew 1996), the crayfish (Krasne and Teshiba 1995) and in the leech (Burrell and Sahley 1998). These intrinsic and extrinsic habituating processes may be independent of each other, so that the disruption of one inhibitory process will not affect the other, allowing the unaffected process to compensate during habituation training.

The effect of 5-HT depletion on $\mathrm{S}_{\mathrm{N}}$ habituation may be explained by the following model of nonassociative learning that incorporates both intrinsic and extrinsic mechanisms of sensitization and habituation. A diagrammatic form of this model is presented in Figure 3. Normally during habituation training, both intrinsic and extrinsic inhibitory processes contribute to the behavioral decrement (Fig. $3 \mathrm{~A})$. In situations where intrinsic sensitization is induced, the intrinsic inhibitory process may be blocked or overridden by the physiological processes that support intrinsic sensitization without affecting the inhibitory components that contribute to extrinsic habituation (Fig. 3B). Even though the $S_{N}$ may share many of the same sensory neurons with the $S_{T}$, habituation can still occur at the $S_{N}$ because it is still affected by extrinsic habituation processes, which compensates for the lack of the intrinsic habituation. This would explain why habituation is prevented at the $\mathrm{S}_{\mathrm{T}}$, the site that is actually receiving repeated stimuli, while habituation at a nearby novel site $\left(\mathrm{S}_{\mathrm{N}}\right)$ can still occur. If this extrinsic habituating process is at least partially 5-HT-dependent, then 5-HT-depleted animals that have also undergone intrinsic sensitization will be unable to express habituation at the $\mathrm{S}_{\mathrm{N}}$. This is because, in addition to having the intrinsic inhibitory processes blocked by intrinsic sensitization, the proposed 5-HT-dependent extrinsic habituating mechanism is also disrupted due to 5 -HT depletion (Fig. 3C). Therefore, since both intrinsic and extrinsic habituating processes are disrupted, habituation is prevented for $\mathrm{S}_{\mathrm{N}}$-elicited responses. One would not expect to have observed this putative 5-HT-dependent component of habituation in previous leech 5-HT depletion experiments because intrinsic sensitization was not induced during those experiments. Therefore, the intrinsic component of habituation was not blocked and could compensate for the disruption of the 5-HTdependent extrinsic inhibitory process.

The possibility that 5-HT may contribute to extrinsic habituation does not contradict the established role of 5-HT in extrinsic sensitization. It simply suggests that 5-HT may have excitatory effects relevant to sensitization on some shortening-related neurons and inhibitory effects relevant to habituation on others. That a single neuromodulator may have multiple and even opposing effects on

$$
\begin{array}{lllllllllllllll} 
& E & A & R & N & I & N & G & \boldsymbol{Q} \\
\mathbf{5 1 5} & M & E & M & O & R & Y
\end{array}
$$


Burrell and Sahley

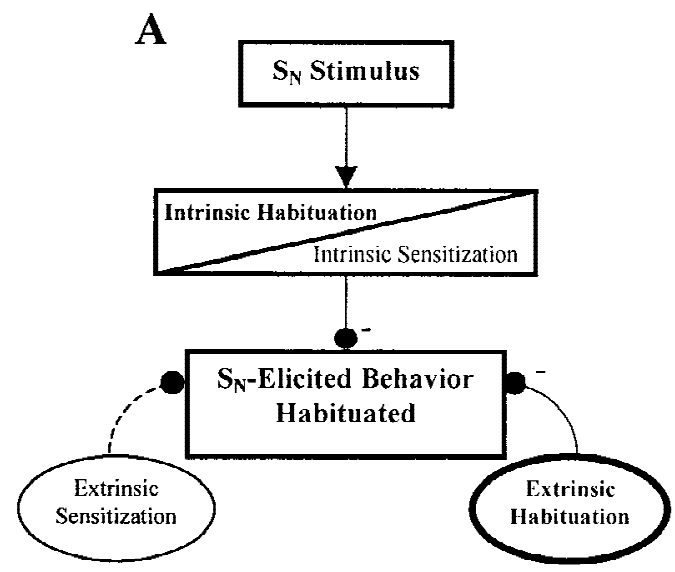

Figure 3: Contribution of intrinsic and extrinsic non-associative learning processes to $S_{N^{-}}$elicited behavior. In this simplified diagram, intrinsic neuromodulation is represented as occurring within the stimulus-response pathway while extrinsic modulatory processes affect the behavior from outside the stimulus-response pathway. These neuromodulatory processes may potentiate $(+)$ or depress $(-)$ the behavior, which ones are active depends on the training conditions. Activated modulatory nodes are indicated in bold and by solid lines connecting them to the behavioral output. Inactivated nodes have normal type set and broken lines showing their connections. The diagrams are not meant to show precise sites of neuroplasticity but are simply demonstrating whether plasticity occurs within (intrinsic) or outside of (extrinsic) the stimulus-response pathway. (A) Habituation. Following normal habituation training, repetitive stimulation generates both intrinsic and extrinsic habituating processes resulting in the re-

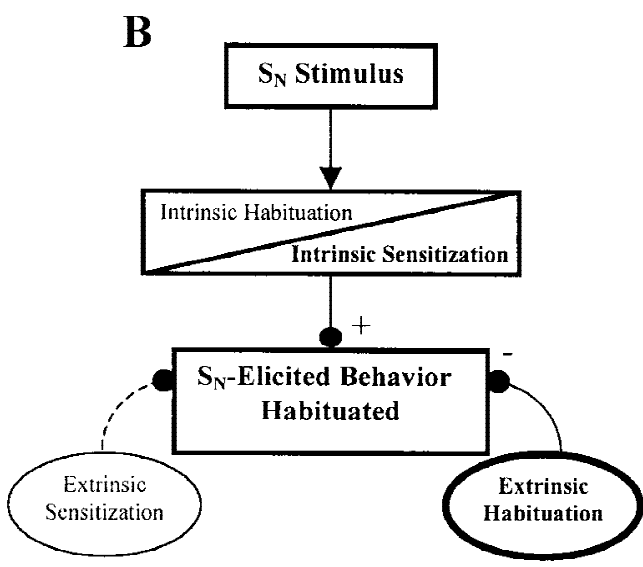
sponse elicited by the $\mathrm{S}_{\mathrm{N}}$. $(B)$ Intrinsic sensitization. Although intrin sic sensitization may block or override intrinsic habituation processes, a nearby stimulus (such as the $S_{N}$ ) may still produce a habituated response due to the influence of the extrinsic habituating process. This extrinsic habituation is the result of repetitive stimulation at the $\mathrm{S}_{\mathrm{T}}$. $(C)$ Intrinsic sensitization with 5 - $\mathrm{HT}$ depletion. The same conditions as $B$, except that extrinsic habituation has been disrupted, potentially due to 5-HT depletion. In the absence of extrinsic habituation, the only modulatory process affecting $S_{N^{-}}$elicited response is intrinsic sensitization. Therefore, the $S_{N^{-}}$elicited response is not depressed despite the repetitive stimulation at the nearby $S_{\mathrm{T}}$.

different neurons contributing to the same behavior has been observed in other animals (Harris-Warrick 1991). In the leech, 5-HT is known to have an inhibitory effect on the mechanosensory pressure cells (LeBmann and Dietzel 1995; Catarsi and Drapeau 1996) and on the R cell (Acosta-Urquidi et al. 1989), while having an excitatory effect on the $S$ cell (B. Burrell, unpubl.), an interneuron known to be critical for extrinsic sensitization of the shortening reflex (Modney et al. 1997). In addition, there is precedent for 5-HT-mediated inhibition of a behavioral reflex, specifically in the restraint-induced inhibition of the crayfish escape response (Glanzman and Krasne 1983; Glanzman and Krasne 1986).

\section{5-HT DEPLETION}

Based on glyoxylic acid staining results, the level of 5-HT depletion in these experiments was similar to previous experiments that have used this depletion procedure (Ehrlich et al. 1992; Sahley 1994). No 5-HT-positive staining was observed in the R cells from the 5-HT-depleted group while the other serotonergic cell bodies were still positively stained. In addition, the R cells from the 5-HT-depleted group exhibited a brown pigmentation that has been observed by other investigators using 5,7DHT to induce 5-HT depletion (Lent 1982; Lent and Dickinson 1984; Kemenes et al. 1989; Ehrlich et al. 1992). Based on quantitative measurements 
by Lent (1984), this depletion procedure results in a near total reduction of 5-HT in the R cell, which translates to a $50 \%$ reduction of $5-\mathrm{HT}$ in a single ganglion.

Although intrinsic sensitization is clearly not dependent on 5-HT-input from the R cell, it may receive serotonergic modulation from other 5-HTcontaining neurons that may not be depleted by 5,7-DHT treatments. Lockery and Kristan (1991) have shown that cells 21 (DL cell) and 61 (VL cell) facilitate the local bending reflex in the leech. For these neurons to play a role in intrinsic sensitization it would have to be shown that they are part of the whole-body shortening neural circuit and that their modulatory effects can be focused on a specific stimulus-response pathway without affecting neighboring pathways. However, it should be noted that even though the soma of the DL and VL cells in 5,7-DHT-treated animals still contain 5-HT, the axons and synapses of these neurons might actually be depleted. The modes of action of 5,7DHT involve (1) the selective absorption of 5,7DHT at the synapses by serotonergic neurons through their 5-HT re-uptake processes, (2) displacement of 5-HT stores within the synapse, and (3) possible inhibition of 5-HT synthesis (Cook and Orchard 1993). As a result, the strongest effects of 5,7-DHT are in the most distal portions of the neuron. Various studies have shown that even when fine processes and release-sites of a neuron are depleted, larger-diameter processes and the cell body may still contain 5-HT (Gadotti et al. 1986; Glanzman et al. 1989; Cook and Orchard 1993). Therefore, it is possible that $5,7-\mathrm{DHT}$ treatment has removed all serotonergic modulation in the leech and that intrinsic sensitization is a completely 5-HT-dependent process. Unfortunately, it has not been possible to visualize the fine processes of 5-HT-containing neurons in the adult leech with either glyoxylic acid or immunocytochemical staining techniques, so this question is left unanswered for now.

\section{CONCLUSIONS}

Traditionally, the physiological mechanisms of non-associative learning have been thought of in terms of intrinsic processes underlying habituation versus extrinsic processes mediating sensitization. Habituation was thought to depend on homosynaptic depression, a decrease in synaptic signaling between neurons within the neurobehavioral circuit (an intrinsic process) (Castellucci et al. 1970;
Walters et al. 1983). Sensitization, on the other hand, was thought to be the result of heterosynaptic facilitation, an increase in synaptic signaling between cells in the neural circuit due to extrinsic neuromodulation (Castellucci and Kanel 1976; Byrne et al. 1978; Hawkins et al. 1981; Ehrlich et al. 1992). Davis and File (1984), and more recently Prescott (1998), have suggested that instead of an exclusive relationship of intrinsic modulation with habituation and extrinsic modulation with sensitization, that both forms of nonassociative learning could utilize intrinsic and extrinsic mechanisms of neural plasticity.

Data from the experiments in this paper indicates that distinct physiological processes mediate intrinsic and extrinsic forms of sensitization, a conclusion that appears to agree with at least one other study (Schnabacher et al. 1996). While the precise physiological process that mediates intrinsic sensitization in the leech is unknown, a strong possibility is that some form of intrinsic neuromodulation plays a role. Intrinsic neuromodulation involves modification of a neural circuit due to the activity of a neuron that is actually part of the circuit being modified. This form of neuromodulation has been observed in neuromuscular circuits (Cropper et al. 1987a,b, 1988), and in central pattern generators (Meyrand et al. 1991; Nusbaum et al. 1992; Katz and Frost 1995). In some cases, intrinsic neuromodulation involves the co-release of both a standard and a modulatory neurotransmitter by the same neuron (Cropper et al. 1987a,b, 1988), while in other cases a single neurochemical acts as both neurotransmitter and neuromodulator (Katz and Frost 1995). Intrinsic neuromodulation is thought to contribute to intrinsic sensitization because the modulatory process is induced by activity of the stimulated circuit and the effect of this modulation is localized to that activated circuit.

Another potential clue as to the cellular basis of intrinsic sensitization is provided by an intriguing study by Jin et al. (1998) and involves the presynaptic activity of $\mathrm{Ca}^{+2} /$ calmodulin-dependent protein kinase II (CaMKII). In this study, transgenic Drosophila expressed an autophosphorylated (and therefore chronically active) form of CaMKII in a selective set of sensory neurons. Habituation training of a leg-withdrawal reflex failed to produce a response decrement in the transgenic flies (Jin et al. 1998). The response pattern looks very similar to what was observed during intrinsic sensitization and it is possible that CaMKII activity in sensory neurons within a stimulus-response pathway plays

$$
\begin{array}{lllllllllllllll} 
& E & A & R & N & I & N & G & \boldsymbol{Q} \\
\mathbf{5 1 7} & M & E & M & O & R & Y
\end{array}
$$


a role in this process. Given the intrinsic sensitization training protocols developed earlier (Burrell and Sahley 1998) and the fact that its nervous system is amenable to detailed physiological studies, the leech should be very useful in studying the potential role of CaMKII, as well as other physiological processes, in intrinsic sensitization.

The data in these experiments, in addition to results from Burrell and Sahley (1998), clearly show that nonassociative learning is mediated by a variety of processes of neuroplasticity. These processes utilize both intrinsic and extrinsic forms of neuromodulation and may interact with each other (as shown in Fig. 3) to produce the final behavioral output. It is important that further studies are carried out to better understand these mechanisms of neuroplasticity, not simply to examine the physiological mechanisms underlying nonassociative learning, but also to uncover modulatory properties of the nervous system that may contribute to more complex, associative forms of learning.

\section{Acknowledgments}

We thank James Randall Fidler for his careful work in generating 5-HT-depleted and ascorbic acid control animals. We thank Jay Hosler, Mark Stopfer and two anonymous reviewers for their helpful comments during the preparation of this manuscript. This research was supported by National Institute of Health grants F32-NS10065 (B.D.B.) and R01-MH44789 (C.L.S.).

The publication costs of this article were defrayed in part by payment of page charges. This article must therefore be hereby marked "advertisemnet" in accordance with 18 USC section 1734 solely to indicate this fact.

\section{References}

Acosta-Urquidi, J., C.L. Sahley, and A.L. Kleinhaus. 1989. Serotonin differentially modulates two $\mathrm{K}^{+}$currents in the Retzius cell of the leech. J. Exp. Biol. 145: 403-417.

Advocat, C. 1980. Modulation of defensive reflexes in Aplysia californica by appetitive stimulation. Behav. Neural Biol. 28: 253-265.

Bagnoli, P., M. Brunelli, and F. Magni. 1972. A fast conducting pathway in the central nervous system of the leech Hirudo medicinalis. Arch. Ital. Biol. 110: 35-51.

Bashinski, H.S., J.S. Werner, and J.W. Rudy. 1985. Determinants of infant visual fixation: Evidence for a two-process theory. J. Exp. Child Psych. 39: 580-598.

Boulis, N. and C. Sahley. 1998. A behavioral analysis of habituation and sensitization of shortening in the semi-intact leech. J. Neurosci. 58: 4621-4627.

Burrell, B.D. and C.L. Sahley. 1998. Generalization of habituation and intrinsic sensitization in the leech. Learn. \& Mem. 5: 405-419.

Byrne, J.H., V.F. Castellucci, and E.R. Kandel. 1978. Contribution of individual mechanoreceptor neurons mediating defensive gill-withdrawal in Aplysia. J. Neurophysiol. 41: 418-431.

Castellucci, V.F. and E.R. Kandel. 1976. Presynaptic facilitation as a mechanism for behavioral sensitization in Aplysia. Science 194: 1176-1178.

Castellucci, V.F., H. Pinsker, I. Kupferman, and E.R. Kandel. 1970. Neuronal mechanisms of habituation and dishabituation of the gill-withdrawal reflex in Aplysia. Science 167: 1745-1748.

Catarsi, S. and P. Drapeau. 1996. Modulation and selection of neurotransmitter responses during synapse formation between identified leech neurons. Cell Mol. Neurobiol. 16: 699-713.

Cook, H. and I. Orchard. 1993. The short term effects of 5,7-dihydroxytryptamine on the peripheral serotonin stores in Rhodnius prolixus and their long-term recovery. Insect Biochem. and Mol. Biol. 23: 895-904.

Cropper, E.C., P.E. Lloyd, W. Reed, R. Tenenbaum, I .Kupferman, and K.R. Weiss. 1987a. Multiple neuropeptides in cholinergic motor neurons of Aplysia: Evidence for modulation intrinsic to the motor circuit. Proc. Natl. Acad. Sci. 84: 3486-3490.

Cropper, E.C., R. Tenenbaum, M.A.G. Kolks, I. Kupferman, and K.R. Weiss. 1987b. Myomodulin: A bioactive neuropeptide present in an identified cholinergic motor neuron of Aplysia. Proc. Natl. Acad. Sci. 84: 5483-5486.

Cropper, E.C., M.W. Miller, S.F. Vilim, R. Tenenbaum, I. Kupferman, and K.R. Weiss. 1988. Buccalin is present in the cholinergic motor neuron B16 of Aplysia and it depresses accessory radula closer muscle contractions evoked by stimulation of B16. Brain Res. 512: 175-179.

Davis, M. 1989. Sensitization of the acoustic startle response by footshock. Behav. Neurosci. 103: 495-503.

Davis, M. and S.E. File. 1984. Intrinsic and extrinsic mechanisms of habituation and sensitization: Implications for the design and analysis of experiments. In Habituation sensitization, and behavior. (ed. H.V.S. Peeke and L.F. Petrinovich), pp. 287-323. Academic Press, New York, NY.

Ehrlich, J., N. Boulis, T. Karrer, and C.L. Sahley. 1992. Differential effects of serotonin depletion on sensitization and dishabituation. J. Neurobiol. 23: 270-279.

Erber, J. and K. Schildberger. 1980. Conditioning of an antennal reflex to visual stimuli in bees (Apis mellifera L.). J. Comp. Physiol. 135: 217-225.

Gadotti, D., L.G. Bauce, K. Lukowiak, and A.G.M. Bulloch.

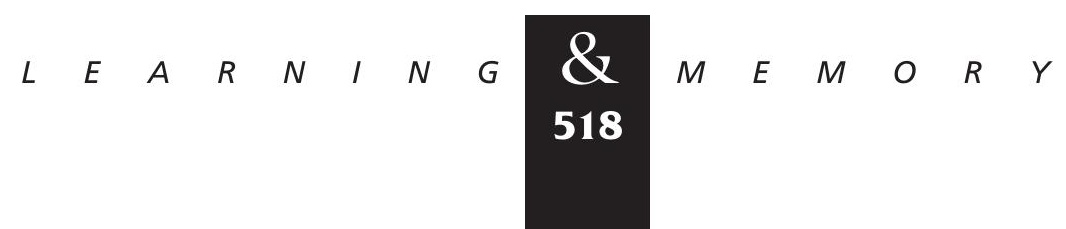


1986. Transient depletion of serotonin in the nervous system of Helisoma. J. Neurobiol. 17: 431-447.

Glanzman, D. and F.B. Krasne. 1983. Serotonin and octopamine have opposite modulatory effects on the crayfish's LG escape reaction. J. Neurosci. 3: 2263-2269.

Glanzman, D.L. and F.B. Krasne. 1986. 5,7-Dihydroxytryptamine lesions of crayfish serotonin-containing neurons: Effect on the lateral giant escape reaction. J. Neurosci. 6: 1560-1569.

Glanzman, D.L., S.L. Mackey, R.D. Hawkins, A.M. Dyke, P.E. Lloyd, and E.R. Kandel. 1989. Depletion of serotonin in the nervous system of Aplysia reduces the behavioral enhancement of gill withdrawal as well as the heterosynaptic facilitation produced by tail shock. J. Neurosci. 9: 4200-4213.

Goldberg, J.I. and K. Lukowiak. 1984. Transfer of habituation in Aplysia: Contribution of heterosynaptic pathways in habituation of the gill-withdrawal reflex. J. Neurobiol. 15: 395-411.

Groves, P.M., D. Lee, and R.F. Thompson. 1970. Effects of stimulus frequency and intesity on habituation and sensitization in acute spinal cat. Physiol. Behav. 4: 383-388.

Harris-Warrick, R.M. 1991. Modulation of neural networks for behavior. Annu. Rev. Neurosci. 14: 39-57.

Hawkins, R.D., V.F. Castellucci, and E.R. Kandel. 1981. Interneurons involved in mediation and modulation of gill-withdrawal reflex in Aplysia II. Identified neurons produce heterosynaptic facilitation contributing to behavioral sensitization. J. Neurophys. 45: 315-328.

Hawkins, R.D., E.R. Kandel, and S.A. Siegelbaum. 1993. Learning to modulate transmitter release: Themes and variations in synaptic plasticity. Annu. Rev. Neurosci. 16: $625-665$.

Hinde, R.A. 1970. Behavioral habituation. In Short-term changes in neural activity and behavior (ed. G. Horn and R.A. Hinde), pp. 3-40. Cambridge University Press, London, UK.

Hitchcock, J.M., C.B. Sananes, and M. Davis. 1989. Sensitization of the startle reflex by footshock: Blockade by lesions of the central nucleus of the amygdala or its efferent pathway to the brainstem. Behav. Neurosci. 103: 509-518.

Jin, P., L.C. Griffith, and R.K. Murphey. 1998. Presynaptic

calcium/camodulin-dependent kinase II regulates habituation of a simple reflex in adult Drosophila. J. Neurosci. 18: $8955-8964$.

Katz, P.S. and W.N. Frost. 1995. Intrinsic neuromodulation in the Tritonia swim CPG: Serotonin mediates both neuromodulation and neurotransmission by the dorsal swim interneurons. J. Neurophys. 74: 2281-2294.
Kemenes, G.Y., K. Elekes, L. Hripi, and P.R. Benjamin. 1989. A comparison of four techniques for mapping the distribution of serotonin and serotonin-containing neurons in fixed and living ganglia of the snail, Lymnaea. J. Neurocytol. 18: 192-208.

Krasne, E.G. and T.M. Teshiba. 1995. Habituation of an invertebrate escape refelx due to modulation by higher centers rather that local events. Proc. Nat. Acad. Sci. 92: 3362-3366.

Kupferman, I. and H. Pinsker. 1968. A behavioral modification of the feeding refelx in Aplysia californica. Commun. Behav. Biol. A. 2: 13-17.

Lent, C.M. 1982. Fluorescent properties of monoamine neurons following glyoxylic acid treatment of intact leech ganglia. Histochemistry 75: 77-89.

Lent. C.M. 1984. Quantitative effect of a neurotoxin upon serotonin levels within tissue compartments of the medicinal leech. J. Neurobiol. 15: 309-323.

Lent, C.M. and M. Dickinson. 1984. Retzius cells retain functional membrane properties following 'ablation' by the neurotoxin 5,7-DHT. Brain Res. 300: 171-176.

Lessman, V. and I.D. Dietzel. 1995. Two kinetically distinct

5-hydroxytryptamine-activated $\mathrm{Cl}^{-}$conductances at Retzius P-cell synapses of the medicinal leech. J. Neurosci. 15: 1496-1505.

Lockery, S.R. and W.B.J. Kristan. 1991. Two forms of sensitization of the local bending reflex of the medicinal leech. J. Comp. Physiol. A 168: 165-177.

Macagno, E.R. and R.R. Stewart. 1987. Cell death during gangliogenesis in the leech: Competition leading to death of PMS neurons has both random and nonrandom components. J. Neurosci. 7: 1911-1918.

Meyrand, P., J. Simmers, and M. Moullins. 1991. Construction of a pattern-generating circuit with neurons of different networks. Nature 351: 60-63.

Muller, K.J., J.G. Nicholls, and G.S. Stent. 1981. Neurobiology of the leech. Cold Spring Harbor Laboratory, Cold Spring Harbor, NY.

Nusbaum, M.P., J.M. Weimann, J. Golowasch, and E. Marder. 1992. Presynaptic control of modulatory fibers by their neural network targets. J. Neurosci. 12: 2706-2714.

Prescott, S.A. 1998. Interactions between depression and facilitation within neural networks: Updating the dual-process theory of plasticity. Learn. \& Mem. 5: 446-466.

Prescott, S. and R. Chase. 1996. Two types of plasticity in the tenacle withdrawal reflex of Helix aspera are dissociated by tissue location and response measure. J. Comp. Physiol. A 179: 407-414.

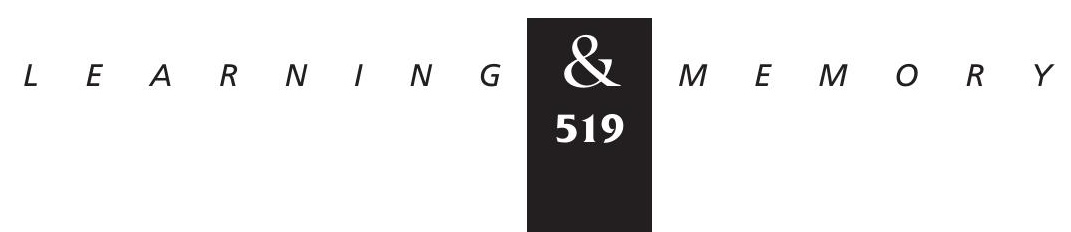




\section{Burrell and Sahley}

Rankin, C.H. and T.J. Carew. 1988. Dishabituation and sensitization emerge as separate processes during development in Aplysia. J. Neurosci. 8: 197-211.

Razran, G. 1971. Mind in Evolution: An East-West Synthesis of Learned Behavior and Cognition. Houghton Mifflin Company, Boston, MA.

Sahley, C.L. 1994. Serotonin depletion impairs but does not eliminate classical conditioning in the leech, Hirudo medicinalis. Behav. Neurosci. 108: 1043-1052.

Schanbacher, A., M. Koch, P.K.D. Pilz, and H.-U. Schnitzler. 1996. Lesions of the Amygdala do not affect the enhancement of the acoustic startle response by background noise. Physiol. Behav. 60: 1341-1346.

Stopfer, M. and T.J. Carew. 1996. Heterosynaptic facilitation of tail sensory neuron synaptic transmission during habituation in tail-induced gill and siphon withdrawal reflexes of Aplysia. J. Neurosci. 16: 4933-4948.

Thompson, R.F., P.M. Groves, T.J. Teyler, and R.A. Roemer. 1973. A dual-process theory of habituation: Theory and behavior. In Habituation. (ed. H.V.S. Peeke and M.J. Herz), pp. 239-271. Academic Press, Orlando, FL.

Walters, E.T., J.H. Byrne, T.J. Carew, and E.R. Kandel. 1983. Mechanoafferent neurons innervating tail of Aplysia: I.

Response properties and synaptic connections. J. Neurophys. 50: 1522-1542.

Zakharov, I.S. and P.M. Balaban. 1987. Neural mechanisms of age-dependent changes in avoidance behavior of the snail Helix lucorum. J. Neurosci. 23: 721-729.

Received July 1, 1999; accepted in revised form August 17, 1999. 


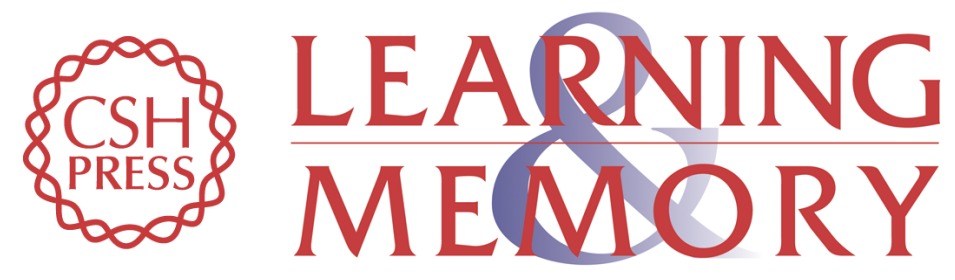

\section{Serotonin Depletion Does Not Prevent Intrinsic Sensitization in the Leech}

Brian D. Burrell and Christie L. Sahley

Learn. Mem. 1999, 6:

Access the most recent version at doi:10.1101//m.6.5.509

References This article cites 43 articles, 17 of which can be accessed free at: http://learnmem.cshlp.org/content/6/5/509.full.html\#ref-list-1

License

Email Alerting Receive free email alerts when new articles cite this article - sign up in the box at the Service top right corner of the article or click here. 